\title{
Conhecimento e Atitudes sobre o Câncer de Próstata no Brasil: Revisão Integrativa
}

https://doi.org/10.32635/2176-9745.RBC.2018v64n4.206

\author{
Knowledge and Attitudes about Prostate Cancer in Brazil: Integrative Review \\ Conocimiento y Actitudes sobre el Cáncer de Próstata en Brasil: Revisión Integrativa
}

Francine Paz Gehres Krüger'; Gustavo Cavalcanti²

Resumo

Introduçáo: $\mathrm{O}$ câncer de próstata está entre as doenças crônicas não transmissíveis que mais afetam os idosos, sendo a idade um fator relevante para esse agravo. É o segundo tipo de câncer de maior prevalência na população masculina, tornando-se um problema de saúde pública. Para tanto, a prevenção e o diagnóstico ficam comprometidos pela baixa procura dos homens ao serviço de saúde. Objetivo: Identificar os obstáculos para a realização do exame de próstata entre os homens. Método: Revisão integrativa da literatura realizada nas bases de dados da BVS, Lilacs, Medline e BDENF. Foram critérios de inclusão: artigos científicos que retratam as ações públicas direcionadas ao câncer de próstata, indexados na base de dados da BVS, publicados em língua portuguesa, inglesa e em espanhol, disponíveis on-line na íntegra. Resultados: Foram encontradas 68 publicaçóes na base de dados Medline, 62 na Lilacs e na BDENF 17, totalizando 147 artigos que poderiam ter relação com o tema da pesquisa. Após leitura dos resumos, desse total, 139 artigos não possuíam relação direta com o estudo, estavam indexados em mais de uma base de dados ou estavam duplicados, totalizando oito artigos publicados para análise. Discussáo: Após leitura interpretativa, emergiram três categorias: dificuldades políticas organizacionais para a prevenção do câncer de próstata, dificuldades socioculturais para prevenção do câncer de próstata e estratégias para prevenção desse câncer. Conclusáo: É necessário desenvolver a prevenção com mais naturalidade, a partir de informaçóes claras e eficazes pelos meios de comunicação.

Palavras-chaves: Neoplasias da Próstata; Saúde do Homem; Adulto; Idoso.

\begin{abstract}
ABSTRACT

Introduction: Breast cancer is the most common type among women in the world and in Brazil, it is part of a heterogeneous group of diseases, thus having different signs and behavior. Possible treatments include surgical approaches, chemotherapy, radiotherapy, endocrine therapy, and target therapy. The surgical procedure can lead to several complications and consequences for the patient, so a follow-up process is necessary after this approach, exercises are fundamental to return functionality and quality of life, but there is divergence in the literature regarding the moment of beginning of the execution of the same. Objective: To perform a systematic literature review, present studies and analyze the impact of the release of immediate versus late exercises, after onco-mammary surgeries, without associated plastic intervention. Method: This is a systematic literature review. To perform the same, a search was made in the database of PubMed, PEDro and SciELO databases using the descriptors: rehabilitation, breast neoplasms, prospective period, prospective care, exercise and their respective ones in the Portuguese language, being these, rehabilitation, breast cancer, postoperative period, postoperative care and exercise. Results: For the accomplishment of this study, 48 articles were found, being 12 relevant to the review. Conclusion: The studies are divergent, but a literary update emphasizes immediate postoperative exercises of onco-mammary surgeries can be used without increasing the incidence of complications.

Key words: Exercise Therapy; Breast Neoplasms; Postoperative Care; Review.
\end{abstract}

\section{Resumen}

Introducción: El cáncer de mama es el tipo más común entre las mujeres en el mundo y en Brasil, forma parte de un grupo heterogéneo de enfermedades, teniendo así signos y comportamiento distintos. Los tratamientos posibles son abordajes quirúrgicos, quimioterapia, radioterapia, endocrinoterapia y terapia objetivo. El procedimiento quirúrgico puede llevar a diversas complicaciones y consecuencias para la paciente, por lo que es necesario un proceso de seguimiento después de este enfoque, ejercicios son fundamentales para devolver funcionalidad y calidad de vida, pero existe divergencia en la literatura en relación al momento de início de la sua ejecución. Objetivo: Realizar una revisión sistemática de la literatura, presentar estudios y analizar el impacto de la liberación de ejercicios inmediatos versus tardíos, después de cirugías onco-mamarias, sin intervención plástica asociada. Método: Se trata de un estudio de revisión sistemática de la literatura. Para la implementación se trataba de una encuesta en la orilla de las bases de datos PubMed, SciELO, PEDro y utilizando las siguientes palabras clave: rehabilitación, neoplasias de la mama, del período prospectivo, de cuidado prospectivo, de ejercicio y su en portugués, siendo éstas, la rehabilitación, el cáncer de mama, período postoperatorio, cuidados postoperatorios y ejercicio. Resultados: Para la realización de este estudio se encontraron 48 artículos, siendo 12 relevantes a la revisión. Conclusión: Los estudios se presentan divergentes, pero se observa una actualización literaria que enfatiza que los ejercicios inmediatos en el postoperatorio de cirugías onco-mamarias pueden ser empleados sin traer aumento en la incidencia de complicaciones.

Palabras clave: Terapia por Ejercicio; Neoplasias de la Mama; Cuidados Posoperatorios; Review.

\footnotetext{
1 Universidade de Passo Fundo/Hospital de Clínicas de Passo Fundo/Prefeitura Municipal de Passo Fundo. Passo Fundo (RS), Brasil. Orcid iD: https://orcid.org/00000002-0335-2760

${ }^{2}$ Universidade de Passo Fundo/Hospital de Clínicas de Passo Fundo/Prefeitura Municipal de Passo Fundo. Passo Fundo (RS), Brasil. Orcid iD: https://orcid.org/00000002-2280-6609

Endereço para correspondência: Francine Paz Gehres Krüger. Universidade de Passo Fundo, Campus II. Rua Teixeira Soares, 817 - Centro. Passo Fundo (RS), Brasil. CEP 99010-080. E-mail:francinegehres@gmail.com
} 


\section{INTRODUÇÃO}

A população idosa vem crescendo de modo significativo; segundo o IBGE, a previsão para 2050 é de 66,5 milhões de pessoas com mais de 60 anos de idade ${ }^{1}$. Nota-se, portanto, que o envelhecimento é compreendido como um processo constituidor da natureza humana, ocorrendo uma diminuição na funcionalidade dos indivíduos. Porém, os fatores estressores, como doenças, acidentes e sobrecarga emocional, acarretam condiçóes patológicas que requerem assistência ${ }^{2}$. Entre as morbidades, as doenças crônicas não transmissíveis $(\mathrm{DCNT})^{2}$ são responsáveis por cerca de $70 \%$ das mortes no mundo 3 .

O câncer está entre as DCNT que mais afetam os homens, em especial, o câncer de próstata, no qual a idade é um fator relevante para tal agravo, aumentando sua incidência na quinta década de vida ${ }^{4,5}$.

Sabe-se que a próstata é uma glândula que faz parte do sistema reprodutivo masculino. Situa-se abaixo da bexiga, envolvendo a uretra e na frente do reto. A glândula prostática possui a função de eliminar a urina que se acumula na bexiga e é também responsável pela produção do esperma que conduz os espermatozoides pelo canal uretral durante $o$ ato sexual ${ }^{6,7}$.

$\mathrm{Na}$ fase inicial do câncer de próstata, ele surge de forma silenciosa, às vezes assintomático, podendo apresentar-se como o crescimento benigno da próstata e como sintoma apenas dificuldade ao urinar. Porém, na fase avançada, pode revelar dor óssea, dificuldade ao urinar ou, na pior das hipóteses, caracterizar-se por infecção generalizada ou insuficiência renal ${ }^{6}$.

Para a Organização Mundial da Saúde (OMS), a estratégia para detecção precoce compreende o diagnóstico precoce, quando apresenta sinais iniciais da doença, e a que não apresenta nenhum sintoma, que é o rastreamento. Os melhores métodos para a investigação são os exames de toque retal e o Prostate-Specific Antigens (PSA) ${ }^{5,8}$.

O Ministério da Saúde, segundo a Portaria no 1.944, de 27 de agosto de $2009^{9}$, propóe que a Política Nacional de Atenção Integral à Saúde do Homem (PNAIHS) ${ }^{10}$, que busca promover a melhoria das condiçôes de saúde da população masculina no Brasil, contribua para redução da morbidade e mortalidade dessa população e facilite o acesso às açôes e aos serviços de assistência integral à saúde. Além disso, pretende organizar, implantar, qualificar e humanizar, em todo o território brasileiro, a atenção integral à saúde do homem.

O câncer de próstata, conforme afirmado anteriormente, constitui-se como um problema de saúde pública, sendo o segundo tipo mais prevalente entre a população masculina, atrás apenas do câncer de pele não melanoma. Outrossim, caracteriza-se por apresentar como fatores de risco o histórico familiar e o índice de massa corporal (IMC) elevado ${ }^{4,5}$.

Para o câncer de próstata, um dos propósitos do Ministério da Saúde é a conscientização para ampliar a adesão da população masculina aos serviços existentes, promovendo açôes de esclarecimento sobre a doença, bem como informaçóes quanto aos riscos e benefícios que envolvem o rastreamento dessa patologia ${ }^{11}$.

Porém, a prevenção e o diagnóstico tornam-se comprometidos pela baixa procura dos homens ao serviço de saúde. A mistificação e os aspectos culturais da masculinidade, como o medo, o machismo, a perda da virilidade, fazem-no recuar ou mesmo adiar a prevenção e o diagnóstico precoce ${ }^{12,13}$. Observa-se que a saúde do homem é um problema de grande relevância para a saúde pública; por isso, é necessário um olhar mais atento para o grupo masculino em estudo. Desse modo, essa investigação, a partir da revisão bibliográfica, buscou analisar quais são os obstáculos detectáveis para a realização do exame de próstata entre os homens.

\section{MÉTODO}

Trata-se de uma revisão integrativo-bibliográfica acerca da temática do câncer de próstata. Destaca-se que a revisáo integrativa consiste em uma análise de pesquisas científicas relevantes, a qual possibilita sintetizar determinado conhecimento sobre um assunto específico de investigação ${ }^{14}$.

Para a realização deste estudo, transcorreram-se as seguintes etapas: seleção do tema, estabelecimento dos critérios de inclusão e exclusão do tema, categorização dos estudos, análise dos estudos, interpretaçáo dos resultados e apresentação da pesquisa $a^{14}$.

A busca dos artigos foi realizada por meio da Biblioteca Virtual em Saúde (BVS), que disponibiliza a seçâo específica de ciência da saúde em geral, permitindo o acesso à Literatura Latino-Americana e do Caribe em Ciências da Saúde (Lilacs), à Medical Literature Analysis and Retrieval System Online (Medline) e ao Banco de Dados em Enfermagem (BDENF). A produção disponibilizada eletronicamente possibilita o acesso fácil a estudos recentes sobre a temática.

Os critérios estabelecidos para seleçâo dos artigos foram: artigos científicos publicados em língua portuguesa, inglesa e espanhola nos anos de 2011 a 2017. Para a busca ativa de tais artigos científicos, foram utilizados os seguintes descritores: câncer de próstata, saúde do homem, adulto, idoso e as expressóes equivalentes em inglês e espanhol.

Como critérios de inclusão, foram estabelecidos os artigos científicos que retratam as açôes públicas direcionadas ao câncer de próstata no Brasil, indexados 
na base de dados da BVS, publicados em inglês, espanhol e português, disponíveis na íntegra on-line.

Como critério de exclusão, consideraram-se os artigos não relacionados à temática proposta nesta investigaçáo, publicados em outras línguas que não a língua portuguesa, inglesa e espanhola, não disponíveis on-line na íntegra, publicados anteriormente a 2011 e/ou com outros gêneros discursivos textuais e acadêmicos de apresentação não relacionados a artigo científico.

Em adição ao percurso desta pesquisa, utilizou-se um instrumento de coleta de dados contendo a identificação do artigo científico, com o intuito de responder à pergunta norteadora: Quais os obstáculos para a realização do exame de próstata entre os idosos?

Visando à avaliação prévia dos estudos, foi realizada a leitura dos artigos em sua totalidade. Para tanto, a presente revisão reuniu e sintetizou oito artigos científicos. As interpretaçôes dos resultados encontrados foram organizadas e apresentadas na forma de tabelas, na busca de açôes para o câncer de próstata.

\section{RESULTADOS}

Obteve-se o resultado de 68 publicaçóes na base de dados Medline, 62 na Lilacs, e 17 na BDENF. No cômputo geral, foram 147 artigos que poderiam ter relaçáo com o tema da pesquisa. Após a leitura dos resumos, desse total, 139 artigos não possuíam relação direta com o estudo em pauta, estavam indexados em mais de uma base de dados ou estavam duplicados.
Para refinamento da busca, foi realizado como parâmetro quatro descritores, sendo o "câncer de próstata" o eixo temático principal de investigaçáo, e tendo como foco a identificação da relaçáo existente entre esse descritor e os demais investigados: saúde do homem, adultos e idoso (Figura 1).

Destaca-se também a utilização de filtros que promoveriam maior refinamento com os seguintes descritores: neoplasia de próstata, detecção precoce de câncer, próstata e saúde do homem. O resultado foi mais positivo, pois alcançou o total de 147 artigos científicos.

Houve, ainda, a necessidade de mais um refinamento em relação à duplicidade da indexação e dos descritores utilizados. Sendo assim, obteve-se um número final de oito artigos a serem analisados, pois atendiam aos critérios de inclusão previamente estabelecidos para este estudo.

A partir de então, os artigos foram analisados na íntegra de acordo com as seguintes variáveis destacadas: título, ano de publicação, autor, base de dados e a revista na qual o estudo foi publicado (Quadro 1).

Quanto à categorização dos artigos segundo o periódico, evidencia-se que aquele com maior número de artigos publicados sobre o tema desta pesquisa foi a Revista de Pesquisa: Cuidado é Fundamental, com $62,5 \%$ do total de publicaçóes, seguida pela revista de Enfermagem da Universidade do Estado do Rio de Janeiro (Uerj) com 25\% e pela Revista Gaúcha de Enfermagem com 12,5\% dos artigos publicados. Quanto ao ano da publicação, destaca-se 2013, com 50\% artigos em periódicos diferentes.

Quadro 1. Relação dos artigos incluídos no estudo, 2018

\begin{tabular}{|c|c|c|c|c|}
\hline Autor & Ano & Título & Base de dados & Revista \\
\hline Souza et al. ${ }^{20}$ & 2011 & $\begin{array}{l}\text { Um toque de masculinidade: a } \\
\text { prevenção do câncer de próstata em } \\
\text { gaúchos tradicionalistas }\end{array}$ & Medline & $\begin{array}{l}\text { Rev. Gaúcha } \\
\text { Enfermagem }\end{array}$ \\
\hline Araujo et al. ${ }^{21}$ & 2013 & $\begin{array}{l}\text { As representações sociais de homens } \\
\text { sobre o câncer de próstata }\end{array}$ & Lilacs & $\begin{array}{l}\text { Rev Pesq Cuid } \\
\text { Fundam Online }\end{array}$ \\
\hline Abreu et al. ${ }^{15}$ & 2013 & $\begin{array}{l}\text { Estratégias para a prevenção do câncer } \\
\text { de próstata }\end{array}$ & Lilacs & $\begin{array}{l}\text { Rev Pesq Cuid } \\
\text { Fundam Online }\end{array}$ \\
\hline Costa et al. ${ }^{16}$ & 2013 & $\begin{array}{l}\text { O significado do toque da próstata para } \\
\text { o homem: Enfermeiro na promoção de } \\
\text { saúde }\end{array}$ & BDENF & $\begin{array}{l}\text { Rev Pesq Cuid } \\
\text { Fundam Online }\end{array}$ \\
\hline Silva et al. ${ }^{22}$ & 2013 & $\begin{array}{l}\text { Conhecimentos e práticas sobre } \\
\text { prevenção do câncer de próstata: uma } \\
\text { contribuição para a enfermagem }\end{array}$ & BDENF & Rev Enferm UERJ \\
\hline Lima et al. ${ }^{17}$ & 2014 & $\begin{array}{l}\text { Os meios alternativos de comunicação } \\
\text { como ferramenta para a prevenção do } \\
\text { câncer de próstata }\end{array}$ & BDENF & Rev Enferm UERJ \\
\hline Amthaver $\mathrm{C}^{19}$ & 2016 & $\begin{array}{l}\text { As representações da masculinidade na } \\
\text { adesão do toque retal como prevenção } \\
\text { contra o câncer prostático }\end{array}$ & Lilacs & $\begin{array}{l}\text { Rev Pesq Cuid } \\
\text { Fundam Online }\end{array}$ \\
\hline Solano et al. ${ }^{18}$ & 2017 & $\begin{array}{l}\text { O acesso do homem ao serviço de } \\
\text { saúde na atenção primária }\end{array}$ & Lilacs & $\begin{array}{l}\text { Rev Pesq Cuid } \\
\text { Fundam Online }\end{array}$ \\
\hline
\end{tabular}




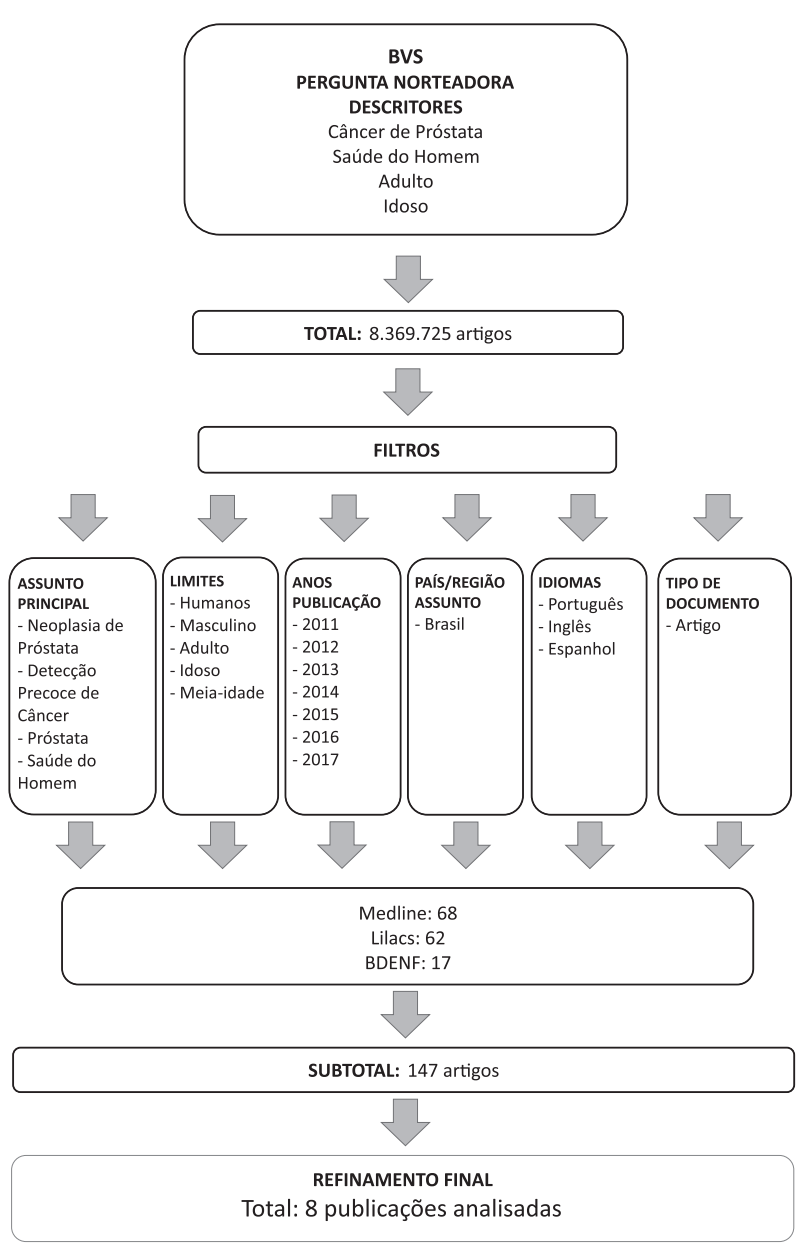

Figura 1. Fluxograma representativo da seleção dos artigos incluídos na revisão integrativa, 2018

Fonte: Base de dados pesquisadas (2018).

Ao analisar o objetivo com o resultado e as respectivas conclusôes dos artigos selecionados, pode-se perceber que todos possuem coerência, sendo de fácil compreensão na interpretaçáo das ideias apresentadas pelos autores.

\section{DISCUSSÃO}

\section{DIFICULDADES POLIITICAS E ORGANIZACIONAIS PARA A PREVENÇÃO DO CÂNCER DE PRÓSTATA}

Nessa categoria, foram selecionadas quatro produçóes científicas que abordaram as principais dificuldades políticas que influenciam na saúde do homem.

Abreu et al. ${ }^{15}$, em seu estudo, tiveram como objetivo identificar as dificuldades do homem para prevenção do câncer de próstata e descrever estratégias para tal a partir de uma revisão sistemática da literatura no período de 2003 a 2010. Sua pesquisa aponta que, entre as dificuldades encontradas pelos homens, destacam-se a precariedade dos serviços públicos ao atendimento e a má qualidade, as filas grandes, a insuficiência de vagas, o horário de atendimento incompatível com a carga horária masculina e a falta de profissionais médicos.

Costa et al. ${ }^{16}$, em sua pesquisa, objetivaram, em primeiro lugar, identificar o significado para os homens sobre o exame clínico de toque digital da próstata para a detecção precoce do câncer, e, em segundo lugar, discutir a atuação do enfermeiro na promoção da saúde dos homens. A abordagem desse estudo foi qualitativa, norteada por formulário semiestruturado. Alguns dos motivos para os homens não buscarem o atendimento nos serviços de saúde apontaram o desconhecimento sobre a política nacional da saúde de atenção integral à saúde do homem, a falta de tempo de se dirigir aos serviços de saúde e os horários aos serviços de saúde serem em horário comercial, considerando que seu horário de trabalho é equivalente e as filas são enormes.

Lima et al. ${ }^{17}$, em seu estudo, analisaram os meios de comunicação como estratégias de promoção da saúde do homem e de prevenção do câncer de próstata. $\mathrm{O}$ estudo tem abordagem quantiqualitativa, buscando a explicação e a compreensão do fenômeno, no qual descreve as variáveis e a dinâmica das relaçóes sociais.

O impedimento na busca por serviços de saúde é ocasionado pelo ambiente majoritamente composto por mulheres nas unidades de saúde, os meios de comunicação não se centram no grupo masculino para incentivar a procura ao serviço de saúde; ou seja, falta divulgação e incentivo na conscientizaçáo de homens sobre o autocuidado, e, quando isso ocorre, a mídia trata o exame de forma pejorativa e constrangedora. Folders, banners e informativos nas campanhas apresentam, certas vezes, palavras de duplo sentido, como tocar e entrar.

Solano et al..$^{18}$ investigaram os aspectos que influenciam no acesso do homem ao serviço de atenção primária. $\mathrm{O}$ estudo exploratório descritivo foi realizado por meio de grupo focal. $\mathrm{O}$ artigo científico mostra que o tempo de espera é um fator impeditivo na busca do serviço, de pouca resolutividade, além de horários incompatíveis com os do homem que trabalha no mesmo horário de seu expediente.

Percebe-se que os autores concordam com as dificuldades da não adesão à saúde pública. Os fatores impeditivos para essa questão foram: serviços de saúde que necessitam integrar os homens aos serviços a partir da capacitação dos profissionais prestadores de atendimento a essa população; além disso, as grandes filas, o atendimento precário, a falta de profissionais e o funcionamento incompatível com o trabalho dos homens foram as principais dificuldades políticas e organizacionais encontradas pelos homens. 
Observando essa categoria, a população masculina demonstra dificuldade de discutir sobre a temática. A PNAIHS ${ }^{10}$ aponta a vulnerabilidade dos homens a DCNT, com prejuízos à qualidade de vida, ainda tendo maior risco e morte em relação a mulheres. Desse modo, a PNAIHS busca compreender as questōes socioculturais e político-econômicas, além de desenvolver açôes de saúde nos diferentes contextos, a fim de reduzir os índices de morbimortalidade por causas previsíveis e evitáveis na população masculina. Porém, os agravos poderiam ser evitados se as políticas direcionadas à prevenção primária fossem realizadas com regularidade.

\section{DIFICULDADES SOCIOCULTURAIS PARA A PREVENÇÃO DO CÂNCER DE PRÓSTATA}

Nessa categoria, foram selecionadas cinco produçôes científicas que abordaram as principais dificuldades socioculturais para a prevençáo do câncer de próstata.

Solano et al. ${ }^{18}$, acerca dessa categoria, ressaltam o quanto a socialização masculina pode fragilizar ou até mesmo afastar os homens do autocuidado, procurando o serviço de saúde apenas quando o adoecimento se torna algo mais grave. Isso se caracteriza pelo fato de que culturalmente a masculinidade é idealizada por força, virilidade e distanciamento do emocional. A resistência masculina, como consequência, gera sobrecarga financeira à sociedade e também sofrimento físico e emocional ao paciente e à sua família pela busca na qualidade de vida.

Abreu et al..$^{15}$, por sua vez, ressaltam que o cuidado à saúde não é visto como uma prática masculina em razão da criação familiar e cultural. O homem é um ser forte e invulnerável, não podendo demostrar sinal de fraqueza. O baixo nível de escolaridade e a situação socioeconômica também são fatores que diminuem as açôes sobre prevenção. $\mathrm{O}$ medo foi um ponto importante no âmbito de vários fatores, como por exemplo, medo de perder um dia de trabalho, medo do toque retal, visto como humilhação e vergonha de ficar exposto a um profissional de saúde - seja homem ou mulher - e o medo de sentir excitação e ereção do pênis.

A pesquisa de Amthauer ${ }^{19}$ teve como objetivo repensar em ações de saúde que transitem nesse território. Afora isso, considerou se tais açóes contemplam todo o universo de sentimentos, dúvidas e incertezas do pensamento masculino quanto ao toque retal. Utilizou-se como método um desenho de ensaio. $\mathrm{O}$ autor faz uma reflexão de que a masculinidade e a feminilidade constroem espaços simbólicos, estruturando a identidade dos sujeitos, as atitudes e as emoçôes a serem seguidas. A virilidade, a agilidade e a eficiência é um requisito forte no discurso do que é ser homem, sendo oposto ao universo feminino, que compóe o comportamento de cuidados à saúde. $\mathrm{O}$ toque retal é algo visto com temor por ser tocado na parte inferior, além da dor tanto simbólica como física associada, como a violação ao ser masculino.

Souza et al. ${ }^{20}$ verificaram a adesão de gaúchos tradicionalistas aos exames preventivos. Identificaram, também, que a cultura gaúcha não influencia no aspecto de não adesão ao exame de toque retal.

Araujo et al. ${ }^{21}$ estudaram as representaçóes sociais de homens sobre o câncer de próstata e a masculinidade frente à doença e suas implicaçôes para a saúde. Essa pesquisa foi exploratória de abordagem qualitativa realizada com homens diagnosticados com câncer de próstata. Os entrevistados associam a próstata como responsável pelo prazer sexual e pela vitalidade do universo masculino, idealizando o homem como um ser forte, viril, dominante do prazer sexual, representaçôes sociais indispensáveis para caracterização de homem. Costa et al. ${ }^{16}$ ressaltam o quanto os homens ainda sentem constrangimento em falar sobre o exame de toque retal.

Os autores supracitados são unânimes ao descrever que a próstata é vista como algo simbólico para o homem, relacionado à virilidade e ao prazer. Portanto, os fatores sociais que permeiam o ser masculino, como virilidade $\mathrm{e}$ força, podem se sobrepor a sentimentos de medo, angústia e receio quando apresentam manifestaçôes clínicas do câncer de próstata, em decorrência da possível percepção do homem de descaracterização do ser masculino frente à sociedade, impossibilitando o indivíduo de buscar o serviço de saúde.

\section{ESTRATÉGIAS PARA A PREVENÇÃO DO CÂNCER DE PRÓSTATA}

Nessa categoria, foram selecionadas quatro produçóes científicas que abordaram as principais estratégias para a prevençáo do câncer de próstata.

Abreu et al. ${ }^{15}$ ressaltam a importância de promover discussōes voltadas ao público masculino não apenas para o toque retal como também para a prevenção de doenças, de modo a prevenir a mortalidade e desmistificar as questôes que permeiam o câncer de próstata. Sobremodo, o exame de rastreamento é a etapa mais importante para a prevençáo, pois, além de ser um método barato, contribui para manutenção da qualidade de vida. Conclui que o profissional enfermeiro deve direcionar as açôes voltadas à desmitificação do medo e do preconceito em relaçáo à doença.

Silva et al. ${ }^{22}$, em sua investigação, objetivaram identificar o conhecimento dos clientes em relação à estratégia de prevenção do câncer de próstata. $\mathrm{O}$ autor propóe que o enfermeiro tenha como um de seus princípios a educaçáo e que proporcione ao público masculino informaçóes relacionadas à saúde do homem, e que possa orientá-lo quanto à prevenção de doenças, aos fatores de riscos e à manutenção de saúde. 
Lima et al. ${ }^{17}$ consideram que os meios de difusão e de informação não atingem, de modo efetivo, a população masculina com suas mensagens, isso ocorre em virtude dos termos pejorativos ou de palavras de duplo sentido, veiculados em mensagens em banners e folders, o que torna a campanha publicitária constrangedora para o público masculino. Ainda salienta a importância dos meios de comunicaçấo alternativos, como a internet, por meio das redes sociais como forma de propagação para a prevenção do câncer de próstata.

Costa et al. ${ }^{16}$ enfatizam a necessidade de discutir sobre a saúde masculina em meios de comunicação, no meio acadêmico científico e no meio das instituiçôes empregadoras, fazendo com que seus funcionários homens sejam estimulados a buscar os serviços de saúde.

Percebe-se, nessa categoria, a ineficiência dos meios de comunicação na transmissão de informações sobre a temática em discussão. Além disso, é fundamental que o enfermeiro estabeleça o vínculo com o indivíduo paciente, de modo a compreender os medos, os anseios, os preconceitos e as dificuldades de entendimento sobre a doença. Em vista disso, poderá favorecer a conscientização dos diretos sobre a saúde, assim como a adesão do homem ao serviço de saúde, podendo reduzir as taxas de morbidade e mortalidade da população masculina.

\section{CONCLUSÃO}

Apesar de existirem políticas públicas para a saúde do homem, evidenciou-se, nos artigos científicos analisados, a dificuldade de o homem aderir às práticas preventivas do câncer de próstata, conforme este estudo se propôs a investigar na revisão bibliográfica sobre essa temática. Embora no Brasil existam campanhas como o "novembro azul”, criada para conscientizar homens acerca do câncer de próstata, os homens costumam dar menos atenção à sua saúde, dificultando o diagnóstico precoce.

Ademais, é necessário enfrentar a prevenção com mais naturalidade, a partir de informaçôes claras e eficazes pelos meios de comunicação e de mídias. Ainda, os profissionais de saúde devem atuar de forma interdisciplinar, pois as questóes que envolvem o câncer de próstata estão para além da fisiopatologia da doença, envolvendo aspectos sociais, culturais e emocionais.

Há, ainda, a necessidade de mais estudos direcionados à temática para melhor compreensão dos aspectos que envolvem a doença, possibilitando a prevenção efetiva, contribuindo para a diminuição dos custos no tratamento da doença e para a melhoraria da qualidade de vida dos homens acometidos pelo câncer de próstata.

\section{CONTRIBUIÇÕES}

Francine Paz Gehres Krüger e Gustavo Cavalcanti contribuíram igualmente no planejamento do estudo, assim como na análise, interpretação dos dados, na elaboração da redação, na revisão crítica e aprovação final da versão a ser publicada.

\section{DECLARAÇÃO DE CONFLITO DE INTERESSES}

Nada a declarar.

\section{FONTES DE FINANCIAMENTO}

Não há.

\section{REFERÊNCIAS}

1. Instituto Brasileiro de Geografia e Estatística. Brasil: uma visão geográfica e ambiental no início do século XXI [Internet]. Rio de Janeiro: IBGE; 2016. [acesso 2018 Ago 09]. Disponível em: https://biblioteca.ibge.gov.br/ visualizacao/livros/liv97884.pdf.

2. Ministério da Saúde (BR). Envelhecimento e saúde da pessoa idosa [Internet]. Brasília, DF: Ministério da Saúde; 2006. [acesso 2018 Ago 09]. (Série A. Normas e Manuais Técnicos); (Caderno de Atenção Básica, n. 19). Disponível em: http://bvsms.saude.gov.br/bvs/publicacoes/ envelhecimento_saude_pessoa_idosa_n19.pdf

3. Word Health Organization. Global Health Estimates 2016: disease burden by cause, age, sex, by country and by region, 2000-2016 [Internet]. Geneva: WHO; 2018. [acesso 2018 Ago 01]. Disponível em: http://www.who.int/healthinfo/ global_burden_disease/estimates/en/index1.html

4. Instituto Nacional de Câncer José de Alencar Gomes da Silva. Estimativa 2018: incidência de câncer no Brasil: síntese de resultados e comentários [Internet]. Rio de Janeiro: INCA; 2018. [acesso 2018 Ago 20]. Disponível em: http://www1.inca.gov.br/estimativa/2018/sintesede-resultados-comentarios.asp

5. Instituto Nacional do Câncer José de Alencar Gomes da Silva. Câncer de próstata: vamos falar sobre isso? [Internet]. Rio de Janeiro: INCA; 2017 [acesso 2018 Ago 20]. Disponível em: http://wwwl.inca.gov.br/inca/ Arquivos/comunicacao/cartilha_cancer_prostata_2017_ final_WEB.pdf

6. Instituto Nacional do Câncer José de Alencar Gomes da Silva. Tipos de câncer: câncer de próstata [Internet]. Rio de Janeiro: INCA; [data desconhecida]. [modificado 2018 Nov 21; acesso 2018 Ago 20]. Disponível em: https:// www.inca.gov.br/tipos-de-cancer/cancer-de-prostata

7. Grossman S. Estrutura e funçấo do sistema geniturinário masculino. In: Porth CG, Grossman S. Fisiopatologia. 9 ed. Rio de Janeiro: Guanabara Koogan; 2016. cap. 51. 
8. Instituto Nacional do Câncer José de Alencar Gomes da Silva. Tipos de câncer: próstata: detecçáo precoce [Internet]. Rio de Janeiro: INCA; [data desconhecida]. [modificado 2018 Nov 21; acessado 20 de agosto]. Disponível em: http:// www2.inca.gov.br/wps/wcm/connect/tiposdecancer/site/ home/prostata/deteccao_precoce

9. Ministério da Saúde (BR). Portaria no 1.944, de 27 de agosto de 2009 [Internet]. [acesso 2018 Ago 20]. Disponível em: http://bvsms.saude.gov.br/bvs/ saudelegis/gm/2009/prt1944_27_08_2009.html

10. Ministério da Saúde (BR). Política nacional de atenção integral a saúde do homem. princípios e diretrizes. Brasília, DF: Ministério da Saúde; 2009.

11. Ministério da Saúde (BR). Lei no 13.045, de 25 de novembro de 2014 [Internet]. [acesso 2018 Ago 20]. Disponível em: http://www.planalto.gov.br/ CCIVIL_03/_Ato2011-2014/2014/Lei/L13045.htm

12. Gomes R. Saúde do homem em debate. Rio de Janeiro: Ed. Fiocruz, 2011. 228 p.

13. Porto SM, Carvalho GB, Fernandes MJM, Ferreira CB. Vivências de homens frente ao diagnóstico de câncer de próstata. Ciências\&Saúde. 2016;9(2):83-89. doi: http:// dx.doi.org/10.15448/1983-652X.2016.2.22225.

14. Souza MT, Silva MD, Carvalho R. Revisão integrativa: o que é e como fazer. Einstein. 2010;8 (1):102-6. doi: http://dx.doi.org/10.1590/s1679-45082010rw1134

15. Abreu AS, Cruz ACA, Cortez EA, Pereira FS, Nascimento RMS. Estratégias para a prevenção do câncer de próstata. Rev Pesq Cuid Fundam Online. 2013;5(2):3795-07.

16. Costa TB, Moura VF. O significado do toque da próstata para o homem: enfermeiro na promoção de saúde. Rev Pesq Cuid Fundam Online. 2013;5(4):537-546. doi: http:// dx.doi.org/10.9789/2175-5361.2013.v5i4.537-546.

17. Lima B, Martins ERC, Ramos RCA, Costa CMA, Clos AC, Ferreira JIC. Os meios alternativos de comunicação como ferramenta para a prevenção do câncer de próstata. Rev Enferm UERJ. 2014; 22(5):656-662.

18. Solano LC, Bezerra MAC, Medeiros RS, Carlos EF, Carvalho FPB, Miranda FAN. O acesso do homem ao serviço de saúde na atenção primária. Rev Pesq Cuid Fundam Online. 2017;9(2):302-308. doi: http://dx.doi. org/10.9789/2175-5361.2017.v9i2.302-308.

19. Amthauer C. As representaçôes da masculinidade na adesão do toque retal como prevenção contra o câncer prostático. Rev Pesq Cuid Fundam Online. 2016;8(3):4733-4737. doi: http://dx.doi.org/10.9789/2175-5361.2016. v8i3.4733-4737.

20. Souza LM, Silva MP, Pinheiro IS. Um toque de masculinidade: a prevenção do câncer de próstata em gaúchos tradicionalistas. Rev. Gaúcha Enferm. 2011;32(1);151-8.

21. Araujo JS, Conceição VM, Silva SED, Santana ME, Vasconcelos EV, Sousa RF. As representaçóes sociais de homens sobre o câncer de próstata. Rev Pesq Cuid Fundam Online. 2013;5(2):3884-93. doi: http://dx.doi. org/10.9789/2175-5361.2013v5n2p3884.

22. Silva ABM, Costa CMA, Spíndola T, Ramos RCA, Martins ERC, Francisco MTR. Conhecimentos e práticas sobre prevenção do câncer de próstata: uma contribuição para a enfermagem. Rev Enferm UERJ. 2013;21(esp.2):785-91. 